\title{
Migration-Based Seismic Trace Interpolation of Sparse Converted Phase Micro-Seismic Data
}

Andrey H. Shabelansky*, Alison Malcolm, Michael Fehler and William L. Rodi, Earth Resources Laboratory, Massachusetts Institute of Technology

\section{SUMMARY}

Multi-component elastic seismic data collected at large offsets have the potential to be used in micro seismic imaging and monitoring. However, types of receiver surface deployments used for micro-seismic monitoring are generally very sparse, and thus the data used for imaging from these receivers cause receiver-migration-operator artifacts that severely contaminate the shallow part of the image. In this study, we present a data-driven method that alleviates these imaging artifacts. The method is based on converted-phase elastic seismic migration and de-migration. We show that despite the spatial aliasing of the recorded data, we are able to suppress the receiver migration-operator artifacts and reconstruct the shallow part of the image. The merit of this approach is that it is elastic, fully data-driven (i.e, independent of source parameters), and does not suffer from migration operator source aliasing, when a small number of shots or micro-seismic events are used. We present a derivation of the method and test it with a synthetic model and a field data set from a geothermal reservoir with abundant natural and induced seismicity.

\section{INTRODUCTION}

Surface micro-seismic acquisitions often have sparse receiver geometries and thus challenge standard seismic imaging by introducing migration operator artifacts, particularly in the shallow subsurface. Conventional data interpolation methods (applied before the imaging step), which are based on the exploitation of structure in a predefined space (i.e, Fourier, wavelet, curvelet) (e.g., Spitz, 1991; Gülünay, 2003; Fomel, 2003; Abma and Kabir, 2006; Naghizadeh and Sacchi, 2007; Herrmann and Hennenfent, 2008; Curry, 2010, and others), cannot typically provide a solution as the data are extremely aliased and noisy. However, imaging using sparse seismic data is still possible for the deeper part of the earth where aliasing is less of a problem. It has also been shown that after the migration step, the image can be iteratively refined and migration artifacts can be suppressed using the so-called least-squares migration (LSQM) approach (e.g., Nemeth et al., 1999; Duquet et al., 2000; Dong et al., 2012), similar to the data mapping approach of Bleistein and Jaramillo (2000). Key components of the LSQM are migration and de-migration steps in which a migrated image is calculated from data and data are calculated from the previously computed image. Wave equation based migration and de-migration (the latter obtained by Born modeling) require source information (i.e., location, mechanism, time-function), which in active source experiment is typically known or can be estimated with high precision. However, in passive-source monitoring the source properties are not directly available, and thus need to be estimated generally with high uncertainty. Thus, standard migration and de-migration from passive sources become cumbersome and uncertain.

To overcome the need to estimate source information, we propose to use the converted phase imaging approach (e.g., Xiao and Leaney, 2010; Shabelansky et al., 2013) for migration, during which we store the back-propagated wavefield in time and then use it in the de-migration in reverse order (i.e., forward) in time as the incident wavefield for Born modeling. Note that for reflection acquisition, the subsurface medium is generally sampled only with the scattered wavefield (i.e., a very small portion of the total wavefield). In the transmission micro-seismic geometry, by contrast, the medium is also sampled with direct waves that contain a large portion of the total wavefield and are recorded at the receivers, despite sparse receiver deployment. Thus, to satisfy the Born approximation (i.e, incident wavefield is of the same order as the total wavefield), it is appropriate to use the back-propagated transmission wavefield (in reverse order in time) as an incident wavefield for Born modeling.

In this paper, we outline a converted phase migration-based interpolation approach and test it with a synthetic model and a field micro-seismic data from a geothermal reservoir in Iceland. We show that despite a very limited number of stations, micro-seismic imaging can be a powerful tool. This procedure can be used as part of an iterative converted phase imaging scheme, as will be discussed in a future publication.

\section{THE ALGORITHM}

The imaging/migration step is the converted-phase elastic reverse time migration (CP-ERTM) (e.g., Shabelansky et al., 2013) which for a single source is given as

$$
I(\underline{x})=\int_{T}^{0} \underline{u}_{p}(\underline{x}, t) \cdot \underline{\ddot{u}}_{s}(\underline{x}, t) d t,
$$

where $\cdot$ is the dot product between vector components, $\underline{x}$ and $t$ are the space (vector) and time coordinates, respectively, and $T$ is the maximum recorded time; it is at the the lower limit of the integral (i.e., the data is propagated backward in time). The wavefields $\underline{\ddot{u}}_{p}$ and $\underline{\ddot{u}}_{s}$ are the P- and S-components of acceleration vector fields decomposed from the isotropic, smooth, elastic wave equation as

$$
\underline{\ddot{u}}_{p}=\alpha^{2} \nabla \nabla \cdot \underline{u} \quad \underline{\ddot{u}}_{s}=-\beta^{2} \nabla \times \nabla \times \underline{u},
$$

where $\nabla, \nabla \cdot$ and $\nabla \times$ are the gradient, divergence and curl, respectively, $\underline{u}(\underline{x}, t)$ is the displacement vector wavefield and $\alpha(\underline{x})$ and $\beta(\underline{x})$ are the $\mathrm{P}$ - and S- wave speeds. The reason for using this imaging approach stems from two important advantages. First, it requires only one elastic propagation to form an image as oppose to the two propagations used in the standard imaging approach (one forward and one backward in time). 


\section{Migration-Based Interpolation of Sparse Converted Phase Data}

The second, more important, advantage is that no source information is needed to form an image, which reduces the imaging uncertainty and computational cost. Note also that we decompose the $\mathrm{P}$ - and $\mathrm{S}$-wavefields using equation 2 rather than using the more computationally efficient Helmholtz decomposition. The reason for this choice stems from the fact that the separated wavefields, using equation 2 , have consistent amplitude polarity, unlike those obtained by the Helmholtz decomposition which require additional treatment for signal amplitude (e.g., Du et al., 2012).

The de-migration step (i.e, the Born modeling) is obtained using the Born approximation of the general form of the elastic wave equation as:

$$
\rho_{0} \delta \underline{\ddot{v}}-\nabla \cdot \mathbf{C}_{\mathbf{0}}: \nabla \delta \underline{v}=\underline{f}
$$

with

$$
\underline{f}=(\nabla \cdot \delta \mathbf{C}: \nabla-\delta \rho) \underline{v}_{0},
$$

where : is the double dot (dyadic) product, $\rho(\underline{x})$ is density, $\mathbf{C}(\underline{x})$ is the fourth order stiffness tensor and $\underline{v}(\underline{x}, t)$ is the forward propagating displacement vector wavefield, each of which are decomposed as $\rho(\underline{x})=\rho_{0}(\underline{x})+\delta \rho(\underline{x}), \mathbf{C}(\underline{x})=\mathbf{C}_{0}(\underline{x})+$ $\delta \mathbf{C}(\underline{x})$ and $\underline{v}(\underline{x}, t)=\underline{v}_{0}(\underline{x}, t)+\delta \underline{v}(\underline{x}, t)$. The subscript 0 refers to a background quantity and $\delta$ denotes a perturbation. The perturbation term, $\nabla \cdot \delta \mathbf{C}(\underline{x}): \nabla-\delta \rho(\underline{x})$, is non-zero at discontinuities in the medium and thus is related to the image; we denote this image as $I_{\text {stack }}(\underline{x})$. The relation between the displacement wavefields in equation 2 and 3 is $\underline{v}_{0}(\underline{x}, t)=\underline{u}(\underline{x}, T-t)$.

Once the wavefields $\delta \underline{v}(\underline{x}, t)$ are modeled, their time records at the receiver positions, $\underline{x}_{o b s}$, are used for CP-ERTM imaging along with the original time records, $\underline{d}^{\text {orig }}\left(\underline{x}_{o b s}, t\right)$, as

$$
\underline{d}^{\text {new }}\left(\underline{x}_{o b s}, t\right)=\gamma \underline{d}^{\text {orig }}\left(\underline{x}_{o b s}, t\right)+\delta \underline{v}\left(\underline{x}_{o b s}, t\right),
$$

where $\underline{d}^{\text {new }}\left(\underline{x}_{o b s}, t\right)$ are new time records and $\gamma$ is a pre-defined weight. The values for $\gamma$ depend primarily on the data illumination and the presence of the converted-phase waves in the data and vary between 0 for no weight to the original data and a very large number, which diminishes the effect of the interpolation.

\section{Description of the Algorithm}

The proposed algorithm consists of the following steps:

1. Back propagate each elastic shot gather, $\underline{d}^{\text {orig }}\left(\underline{x}_{o b s}, t\right)$, in time. During the back-propagation:

(a) Decompose $\underline{u}(\underline{x}, t)$, into P-and S- wavefields using equation 2 .

(b) Construct an image, $I(\underline{x})$, using equation 1.

(c) Store the displacement (or particle-velocity) vector wavefields, $\underline{u}(\underline{x}, t)$.

2. Stack over all images to obtain an image, $I_{\text {stack }}(\underline{x})$.

3. For each shot (or earthquake):

(a) Forward propagate the stored wavefield, $\underline{v}_{0}(\underline{x}, t)=$ $\underline{u}(\underline{x}, T-t)$, multiplied by the image, $I_{\text {stack }}(\underline{x})$, to obtain $\delta \underline{v}(\underline{x}, t)$. (b) Record the time history of the forward-propagated wavefield at the receiver depth, $\delta \underline{v}\left(\underline{x}_{o b s}, t\right)$, and construct the new shot gather, $\underline{d}^{n e w}\left(\underline{x}_{o b s}, t\right)$, using equation 5 .

4. Repeat steps 1(a), 1(b) and 2 with the new shot gathers.

\section{RESULTS}

To examine the proposed approach for interpolation-based CPERTM, we test it with two data sets: a synthetic data set based on the field data and a field data set from the passive source micro-seismic monitoring of a geothermal area in Iceland. The acquisition geometry is transmission for both tests, i.e., the recording stations are placed on the surface and sources are located at depth (n.b., some earthquakes in the field data occurred outside of the imaging/computational region). All elastic wave solutions for migration and de-migration are modeled with a 2D finite-difference solver, using a second order in time staggered-grid pseudo-spectral method with perfectly matched layer (PML) absorbing boundary conditions (Kosloff et al., 1984; Carcione, 1999; Marcinkovich and Olsen, 2003).

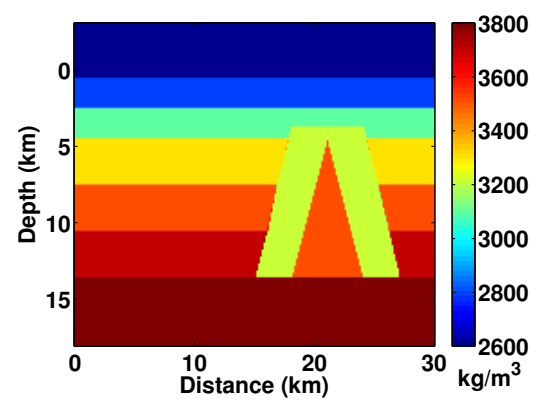

Figure 1: Density, $\rho(\underline{x})$, model used for synthetic tests.

\section{Synthetic Model}

The synthetic model, shown in Figure 1, is the density model which defines the contrast of the medium. The P-and S-wave speeds are constant $4.5 \mathrm{~km} / \mathrm{s}$ and $2.5 \mathrm{~km} / \mathrm{s}$, respectively. The number of grid points in the model is $N_{z}=140$ and $N_{x}=200$, and the spatial increments are $\Delta x=\Delta z=0.15 \mathrm{~km}$. We generate 21 isotropic sources equally distributed at $15.0 \mathrm{~km}$ depth with a horizontal increment of $1.2 \mathrm{~km}$ using a Ricker wavelet with a peak frequency of $6 \mathrm{~Hz}$ and $\Delta t$ of $0.006 \mathrm{~s}$. The data are recorded with two-component receivers that are equally distributed on the surface, $0 \mathrm{~m}$, and span the computational grid. A representative shot gather (Z-component) generated from $(x, z)=(7.8,15) \mathrm{km}$ is shown in Figure2(a), and the CPERTM imaging result using 21 shots is shown in Figure2(b).

Having calculated the elastic seismic data records, we decimate them by about $97 \%$ (see a representative shot gather in Figure 3(a)), leaving only 4 live traces. The reason for this small number stems from the field data example discussed below. Next, we construct an image using the 21 decimated shots (Figure 3(b)). We observe that the image is contaminated with noise due to the data sparsity. Using the obtained image and stored wavefield from back-propagations, $\underline{v}_{0}(\underline{x}, t)$, we 


\section{Migration-Based Interpolation of Sparse Converted Phase Data}

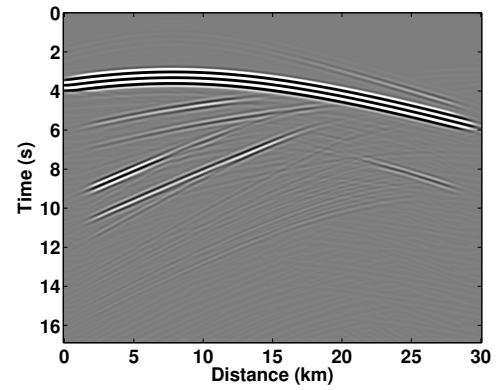

(a)

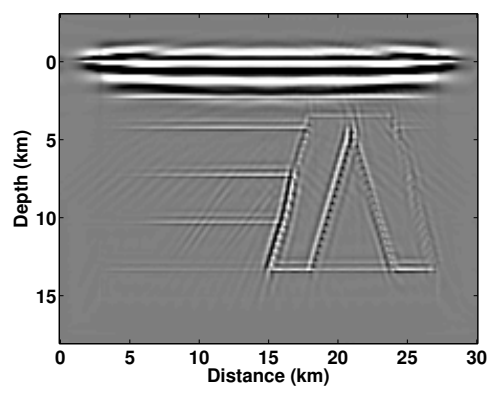

(b)

Figure 2: (a) A representative common shot gather recorded at the surface, $0 \mathrm{~km}$, from a shot at depth $(x, z)=(7.8,15.0) \mathrm{km}$. (b) CP-ERTM imaging result obtained with all 21 shots. Although there are a few artifacts, the structures are well-imaged.

apply Born modeling with a constant density of $3000 \mathrm{~kg} / \mathrm{m}^{3}$ for $\rho_{0}$ (see equation 3). In Figure 3(c) we show the modeled/reconstructed traces for the shot gather shown in Figure 3(a). We observe that although the direct P-wave is clearly absent and the data are somewhat noisy, the missing traces are reconstructed to some degree. To test the quality of the reconstructed data, we add the reconstructed data to the initial data (comprising 4 live traces) with $\gamma=1.8$ (equation 5), and calculate the CP-ERTM image with 21 shots (see Figure 3(d)). In Figure 3(d), we observe that although a number of artifacts remain, many are also removed and the noise level in the shallow part of the image are reduced, without affecting the image quality as compared to Figure 3(b). In this synthetic example the weight, $\gamma$, for the original decimated data is high because most converted waves here are highly dependent on the direct P-wave, which was clearly missing in the Born-modeled data. However, if the conversions are primarily generated by other (non-direct) waves, the weight for the original decimated data can be small or even set to zero. This is illustrated with the field data example.

\section{A field data example from a micro-seismic monitoring}

The second data example is field, passive source, micro-seismic data from a geothermal area in Iceland with abundant natural and induced seismicity. Only four stations along a 2D line are available for 2D imaging. In Figure 4(a), we show a representative common shot gather (Z-component) for these four traces from a single earthquake. The relative position of the traces in the common shot gather corresponds to the relative locations of the stations at the surface, $\underline{x}_{o b s}=\left(x, z_{\text {surface }}=0 \mathrm{~km}\right)$. The

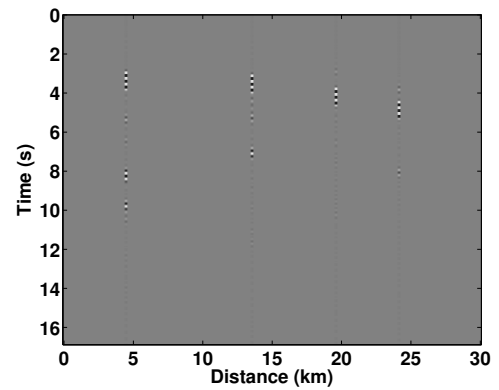

(a)
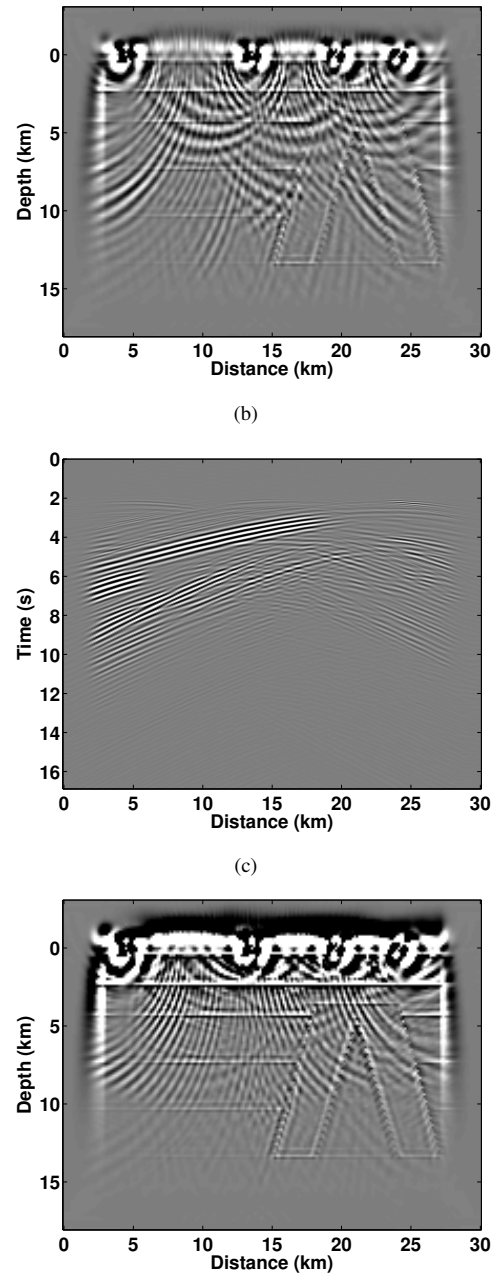

(d)

Figure 3: Same data as shown in Figure 2: (a) shot gather with only 4 live traces (i.e., about 97 percent of original traces were removed), (b) Converted-phase image obtained with 21 shots using only 4 live traces, (c) modeled data using Born modeling, (d) Converted-phase image obtained with 21 modeled shots using $\gamma=1.8$. 


\section{Migration-Based Interpolation of Sparse Converted Phase Data}

extracted time window record of $T=12 \mathrm{~s}$ and $\Delta t=0.005 \mathrm{~s}$ is band-pass filtered between 2 and $12 \mathrm{~Hz}$. The computational grid is $N_{z}=90$ and $N_{x}=300$ and the spatial increments are $\Delta z=0.2 \mathrm{~km}$ and $\Delta x=0.15 \mathrm{~km}$. To construct an image of the geothermal area (using equation 1) we use 32 events (i.e., earthquakes) of moment magnitude between 0.9 and 1.2, and $\mathrm{P}$ - and $\mathrm{S}$-wave speeds, previously estimated by conventional regional-scale travel-time tomography (Tryggvason et al., 2002). In Figure 4(b), we show the CP-ERTM image stacked over all 32 events. We observe (in Figure 4(b)) that although the deeper part of the image is reasonable, the shallow part is completely contaminated.

Now, after having generated a stacked image, $I_{\text {stack }}(\underline{x})$, we use it along with the stored wavefield $\underline{v}_{0}(\underline{x}, t)$, for each event, as input for generating the forward propagating wavefield $\delta v(\underline{x}, t)$ (i.e, Born modeling using equations 3 and 4). The result in Figure 4(c) is the modeled seismic shot gather that corresponds to that shown in Figure 4(a). We observe that the relative amplitude of the traces shown in Figure 4(a) are preserved in Figure 4(c) and additional data are generated at previously empty traces. By migrating only these new 32 seismic shot gathers (without using the original data, $\gamma=0$ ), we obtain the new migrated image that is shown in Figure 4(d). We observe that the deeper part remains almost the same whereas the artifacts in the shallow part are considerably suppressed.

\section{CONCLUSIONS}

In this study, we presented a practical, data-driven, approach for migration-based seismic interpolation of sparse transmission, micro-seismic data using converted-phases. We demonstrated the approach with synthetic and field data using only an extremely small number of stations. We showed that, using this approach, it is possible to image not only the deeper part of the earth but also its shallow part, without any sort of source information. This opens up the possibility of imaging with sparsely recorded micro-seismic data without the artifacts such imaging usually generates in the shallow subsurface.

\section{ACKNOWLEDGMENTS}

We thank ConocoPhillips and the ERL founding members consortium at MIT for funding this work. We also acknowledge Olafur Gudmundsson \& Ari Tryggvason from Uppsala University, Sweden, for providing velocity models and for collaboration with collecting the micro-seismic data

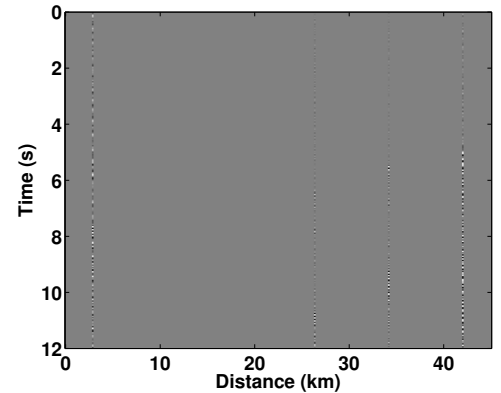

(a)
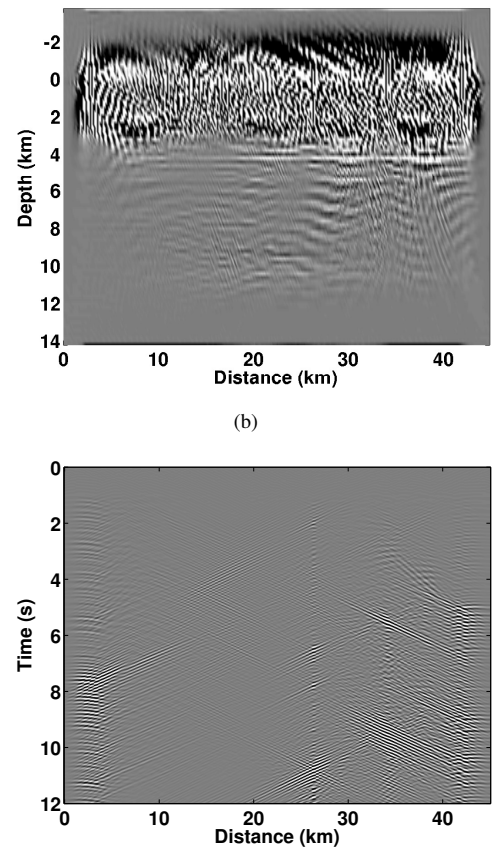

(c)

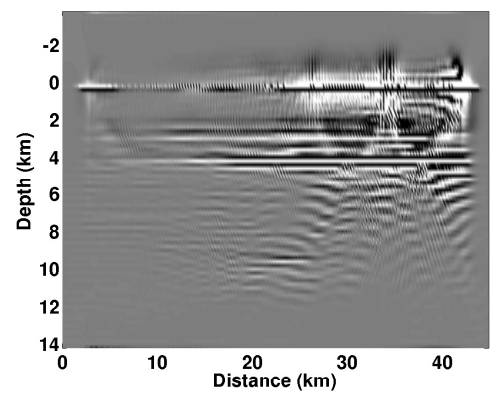

(d)

Figure 4: (a) Seismic traces from four receivers sorted into a common shot gather (Z-component). The data are band-pass filtered with passband 2 and $12 \mathrm{~Hz}$. (b) CP-ERTM image obtained with 32 events (earthquakes). The structure on the right corresponds to the structure of a volcano. (c) Born-modeled common shot gather corresponding to that is shown in (a). (d) CP-ERMT image obtained with the Born-modeled data and the same 32 events as in (b) (using $\gamma=0$ ). The structure of the deeper part of the image is preserved and the artifacts from shallow part of the image are suppressed. 
http://dx.doi.org/10.1190/segam2014-0558.1

\section{EDITED REFERENCES}

Note: This reference list is a copy-edited version of the reference list submitted by the author. Reference lists for the 2014 SEG Technical Program Expanded Abstracts have been copy edited so that references provided with the online metadata for each paper will achieve a high degree of linking to cited sources that appear on the Web.

\section{REFERENCES}

Bleistein, N., and H. Jaramillo, 2000, A platform for Kirchhoff data mapping in scalar models of data acquisition: Geophysical Prospecting, 48, no. 1, 135-161, http://dx.doi.org/10.1046/j.13652478.2000.00178.x.

Carcione, J. M., 1999, Staggered mesh for the anisotropic and viscoelastic wave equation: Geophysics, 64, 1863-1866.

Curry, W., 2010, Interpolation with Fourier-radial adaptive thresholding: Geophysics, 75, no. 6, WB95WB102, http://dx.doi.org/10.1190/1.3500977.

Dong, S., J. Cai, M. Guo, S. Suh, Z. Zhang, B. Wang, and Z. Li, 2012, Least-squares reverse time migration: Towards true amplitude imaging and improving the resolution: 82nd Annual International Meeting, SEG, Expanded Abstracts, doi: 10.1190/segam2012-1488.1.

Du, Q., Y. Zhu, and J. Ba, 2012, Polarity reversal correction for elastic reverse time migration: Geophysics, 77, no. 2, S31-S41, http://dx.doi.org/10.1190/geo2011-0348.1.

Duquet, B., K. J. Marfurt, and J. A. Dellinger, 2000, Kirchhoff modeling, inversion for reflectivity, and subsurface illumination: Geophysics, 65, 1195-1209, http://dx.doi.org/10.1190/1.1444812.

Fomel S., 2003, Seismic reflection data interpolation with differential offset and shot continuation: Geophysics, 68, 733-744, http://dx.doi.org/10.1190/1.1567243.

Gulunay, N., 2003, Seismic trace interpolation in the Fourier transform domain: Geophysics, 68, 355369, http://dx.doi.org/10.1190/1.1543221.

Herrmann, F. J., and G. Hennenfent, 2008, Non-parametric seismic data recovery with curvelet frames: Geophysical Journal International, 173, no. 1, 233-248, http://dx.doi.org/10.1111/j.1365246X.2007.03698.X.

Kosloff, D., M. Reshef, and D. Loewenthal, 1984, Elastic wave calculations by the Fourier method: Bulletin of the Seismological Society of America, 74, no. 3, 875-891.

Marcinkovich, C., and K. Olsen, 2003, On the implementation of perfectly matched layers in a threedimensional fourth-order velocity-stress finite difference scheme: Journal of Geophysical Research, 108, no. B5, doi: 10.1029/2002JB002235.

Naghizadeh, M., and M. D. Sacchi, 2007, Multistep autoregressive reconstruction of seismic records: Geophysics, 72, no. 6, V111-V118, http://dx.doi.org/10.1190/1.2771685.

Nemeth, T., C. Wu, and G. T. Schuster, 1999, Least-squares migration of incomplete reflection data: Geophysics, 64, 208-221, http://dx.doi.org/10.1190/1.1444517.

Shabelansky, A., A. Malcolm, M. Fehler, X. Shang, and W. Rodi, 2013, Converted phase elastic migration velocity analysis : 83rd Annual International Meeting, SEG, Expanded Abstracts, 47324737, http://dx.doi.org/10.1190/segam2013-1265.1. 
Spitz, S., 1991, Seismic trace interpolation in the F-X domain: Geophysics, 56, 785-794, http://dx.doi.org/10.1190/1.1443096.

Tryggvason, A., S. T. Rögnvaldsson, and O. G. Flóvenz, 2002, Three-dimensional imaging of the $P$ - and $S$-wave velocity structure and earthquake locations beneath southwest Iceland: Geophysical Journal International, 151, no. 3, 848-866, http://dx.doi.org/10.1046/j.1365-246X.2002.01812.x.

Xiao, X., and W. Leaney, 2010, Local vertical seismic profiling (VSP) elastic reverse-time migration and migration resolution: Salt-flank imaging with transmitted P-to-S waves: Geophysics, 75, no. 2, S35S49, http://dx.doi.org/10.1190/1.3309460. 